\title{
Assessment of the Pharmacopeial Analytical Methodologies in the Dissolution Test of Enteric-Coated Lansoprazole Preparations
}

\author{
Alaa M. Abu Hammad, Imad I. Hamdan*, and Dina El-Sabawi \\ Faculty of Pharmacy, The University of Jordan, Amman 11942, Jordan
}

e-mail: i.hamdan@ju.edu.jo

\section{ABSTRACT}

The performance of the analytical methodologies recommended by the United States Pharmacopoeia (USP) monograph (1) for dissolution testing of lansoprazole (LPZ) enteric-coated solid dosage forms (capsules/tablets) was critically evaluated. While USP adopts an essentially nonselective UV method, the British Pharmacopoeia (2) recommends a highperformance liquid chromatography (HPLC) method capable of separating the drug from its acid degradation products. For an acid-labile drug such as LPZ, one might think that the nonselective UV method might over- or underestimate the percentage released because the degradation product might have UV absorptivity different from that of the parent drug. We subjected six commercial products in addition to the reference product (G) to analysis according to the USP assay and dissolution recommendations. Products were also subjected to dissolution tests whereby a selective HPLC method was employed for quantifying the percentage release. All products passed USP assay tests. For dissolution, the UV method adopted by USP was more reliable because it indicated the actual percentage released compared with the selective HPLC method, which reflected only the percentage of released LPZ that remained intact (undegraded). Only one product (E) failed to satisfy the USP requirements for dissolution.

KEYWORDS: Lansoprazole; dissolution; acid labile; pharmacopeia; quality.

\section{INTRODUCTION}

ansoprazole (LPZ, shown in Figure 1) is a proton-pump inhibitor that can be given orally and is indicated for the treatment and control of acid-related diseases such as gastro-duodenal ulcers and reflux esophagitis (3). LPZ is known for its chemical instability in acidic media and is, therefore, usually manufactured in the form of enteric-coated tablets or pellets in capsules (4). According to the data presented by Gupta et al. (4), a dramatic increase in the degradation rate of LPZ was observed as the $\mathrm{pH}$ dropped only one $\mathrm{pH}$ unit from 6 to 5 . LPZ has been quantitatively determined in formulations using different

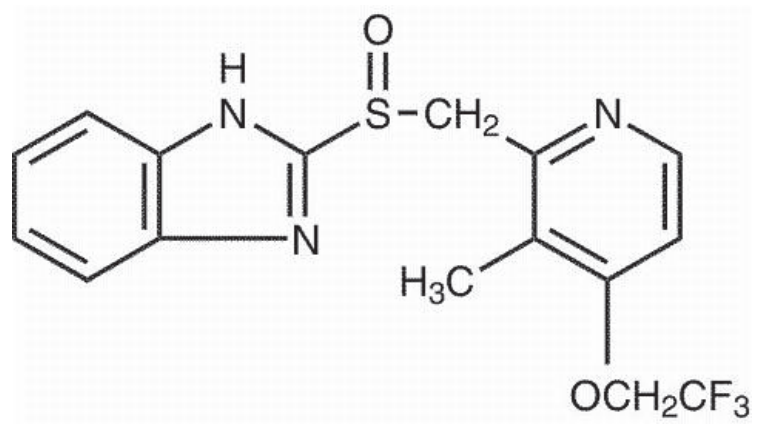

Figure 1. Chemical structure of lansoprazole (LPZ). analytical methods including spectrophotometry, highperformance liquid chromatography (HPLC), and thinlayer chromatography (5-14).

LPZ is an official drug in both the USP (1) and the British Pharmacopoeia (2) where monographs are available for each of the raw material and delayed-release capsules. Both the USP and BP adopt chromatographic HPLC methods that share some parameters (e.g., mobile phase composition employed for assay of capsules), but they differ in others (e.g., the stationary phase chemistry and the use of internal versus external standards). Both the USP and $B P$ require the performance of dissolution testing for the finished products over two stages (acidic and nearly neutral). Dissolution tests commonly employ a UV spectroscopic method because of simplicity of the method and absence of usual interferences from the formulation components. However, in some cases where the drug is acid labile, the degradation might be fast enough so that the resultant degradation products interfere with the simple nonselective UV procedure (15). Such a potentially serious effect on the overall dissolution results has been demonstrated for amoxicillin capsules (16). Concerning dissolution testing of LPZ; the 
USP (1) adopts a UV spectroscopic method while the $B P(2)$ adopts a more selective HPLC method. Because LPZ rapidly degrades in acidic medium, the use of a nonselective UV method could be potentially risky and may lead to inaccurate conclusions about the percentage of drug that is actually released.

This study aims to critically assess the nonselective analytical method recommended by the USP (UV spectroscopy) for dissolution testing of LPZ entericcoated solid preparations in comparison to a selective HPLC method such as that recommended by the $B P$.

\section{EXPERIMENTAL}

\section{Materials and Equipment}

Working standards of LPZ were obtained from the Jordanian Pharmaceutical Manufacturing Company (JPM) with a potency of $99.8 \%$. HPLC grade acetonitrile, trimethylamine, and methanol were obtained from TEDIA (USA). The internal standard (4-ethoxyacetophenone) and other reagents were obtained from Sigma-Aldrich (Germany). All solid dosage forms of LPZ commercially available in the Jordanian market were purchased from local pharmacies (Table 1).

Table 1. Details of the Tested Preparations of Lansoprazole

\begin{tabular}{|c|c|c|l|}
\hline $\begin{array}{c}\text { Commercial } \\
\text { lansoprazole } \\
\text { preparation } \\
\text { code }\end{array}$ & $\begin{array}{c}\text { Dosage } \\
\text { Form }^{a}\end{array}$ & $\begin{array}{c}\text { Batch } \\
\text { Number }\end{array}$ & $\begin{array}{c}\text { Production-Expiry } \\
\text { Dates }\end{array}$ \\
\hline A & tablet & 121286 & $12 / 2012-12 / 2015$ \\
\hline B & capsule & 0413339 & $06 / 2013-06 / 2016$ \\
\hline C & capsule & 679 & $07 / 2012-07 / 2014$ \\
\hline D & capsule & 32018 & $08 / 2013-08 / 2015$ \\
\hline E & capsule & 254022 & $08 / 2012-08 / 2014$ \\
\hline F & capsule & 130428 & $04 / 2013-04 / 2016$ \\
\hline G & capsule & 2 D07C & $05 / 2012-04 / 2014$ \\
\hline
\end{tabular}

${ }^{a}$ All contained $30 \mathrm{mg} / \mathrm{unit}$

A Merck Hitachi HPLC system (model L-7400, Tokyo, Japan) consisting of a UV detector and an isocratic pump was used. Chromatograms were monitored and integrated using Clarity Light software. A typical C18 column $(5 \mu \mathrm{m}, 250 \times 4.6 \mathrm{~mm}$, Thermo Scientific, USA) as recommended by the USP was employed. All spectroscopic measurements were made using 1-cm quartz cells on a Spectroscan 80D, UV-vis spectrophotometer (Biotech Engineering Management Co. Ltd, UK).

\section{Analytical Procedures}

\section{USP Assay Procedure}

The commercial preparations were subjected to the official USP assay test to verify their content before performing dissolution tests. The USP procedure was strictly followed, and a highlight of the major points is described below. The wavelength of detection was $285 \mathrm{~nm}$, and the mobile phase consisted of 1:40:60 triethylamine/acetonitrile/pH 10.5 orthophosphoric acid in water adjusted to $\mathrm{pH} 7$ and pumped at a rate of $1 \mathrm{~mL} /$ min. Test solutions (known as assay preparations) were prepared three times for each commercial product and injected five times onto the HPLC. Standard preparations were made by mixing $25 \mathrm{~mL}$ of standard LPZ solution (3 $\mathrm{mg} / \mathrm{mL}$ in $0.1 \mathrm{M} \mathrm{NaOH} /$ acetonitrile in a ratio of 3:2) with 5 $\mathrm{mL}$ of internal standard solution $(7.5 \mathrm{mg} / \mathrm{mL})$, diluting to a volume of $50 \mathrm{~mL}$, and further diluting $3 \mathrm{~mL}$ of the mixture to $50 \mathrm{~mL}$. Thus, the final standard preparation mixture contained 90 and $45 \mu \mathrm{g} / \mathrm{mL}$ of LPZ and internal standard, respectively, which are the same concentrations expected for the assay preparations.

\section{Details of Dissolution Tests}

Dissolution tests were performed per the USP monograph using Apparatus 2 with a paddle rotation speed set at 75 $\mathrm{rpm}$. Six units from each product were subjected to the dissolution test. Dissolution was performed at two stages for all products, the acid stage followed by the buffer stage. The first acid stage medium consisted of $500 \mathrm{~mL}$ of $0.1 \mathrm{M}$ hydrochloric acid $(\mathrm{HCl})$. Sampling at this stage was made at $60 \mathrm{~min}$ by withdrawing a $25-\mathrm{mL}$ aliquot then proceeding directly to the second buffer stage by adding $425 \mathrm{~mL}$ of buffer concentrate to the remaining $475 \mathrm{~mL}$ acid stage medium so that the total buffer stage volume would be $900 \mathrm{~mL}$. The buffer concentrate was prepared by transferring $65.4 \mathrm{~g}$ of monobasic sodium phosphate, $28.2 \mathrm{~g}$ of sodium hydroxide, and $12 \mathrm{~g}$ of sodium dodecyl sulfate to a suitable container and adding enough water to dissolve, then diluting with water to $4 \mathrm{~L}$. While the USP monograph requires one sample to be taken from the buffer stage at $60 \mathrm{~min}$, in this study samples were obtained at 30,60, and $90 \mathrm{~min}$. The amount of LPZ dissolved in all tests samples was determined by employing the USP-recommended UV spectroscopic method as follows. For the acid stage sample, absorption at the wavelength of maximum absorbance (306 nm) was measured using the acid-stage medium as a blank and compared with a standard of LPZ solution ( $5 \mu \mathrm{g} /$ $\mathrm{mL}$ ) in $0.1 \mathrm{M} \mathrm{HCl}$. For the buffer stage, the absorbance of samples was determined using the difference between absorbances at the wavelengths of 286 and $650 \mathrm{~nm}$ using 
a blank solution composed of acid stage medium and a buffer concentrate adjusted to $\mathrm{pH} 6.8$ (volume ratio 19:17) and compared with a standard solution of LPZ $(23.3 \mu \mathrm{g} /$ $\mathrm{mL}$ ). Five calibration solutions were prepared to contain $1.5-15 \mu \mathrm{g} / \mathrm{mL}$ of $\mathrm{LPZ}$ in $0.1 \mathrm{M} \mathrm{HCl}$ for the acid stage and 6-30 $\mu \mathrm{g} / \mathrm{mL}$ for the buffer stage to ensure linearity of the response in each case.

In addition to the above-detailed UV method, the concentrations of LPZ in dissolution samples (acid or buffer stages) were also determined using an HPLC method. The chromatographic method employed was essentially that recommended by the USP for the assay procedure after being adapted for the dissolution samples as follows. Each 2-mL dissolution sample was mixed with $2 \mathrm{~mL}$ of internal standard solution $(30 \mu \mathrm{g} /$ $\mathrm{mL}$ of 4-ethoxyacetophenone) and diluted to a volume of $10 \mathrm{~mL}$ using USP diluents before it was injected onto the HPLC. Calculation of the dissolved concentration of LPZ was achieved by comparing the peak area ratio of LPZ to the internal standard in the sample and a similarly prepared standard solution.

\section{RESULTS AND DISCUSSION}

\section{Assay Values}

To properly evaluate the characteristic release performance of the different products, the content of LPZ was determined according to the proper assay procedure recommended by the USP. As a part of a system suitability test, the USP requires that the resolution between LPZ and internal standard must be greater than 5 and the relative standard deviation (RSD) of multiple injections less than $2 \%$. Therefore, before any analysis and data collection, we ensured that this essential requirement was achieved and maintained over the period of analysis. A sample chromatogram for the system suitability test resolution solution is shown in Figure 2. The obtained average percentage per label and corresponding RSD values for all tested products are listed in Table 2. Generally, the

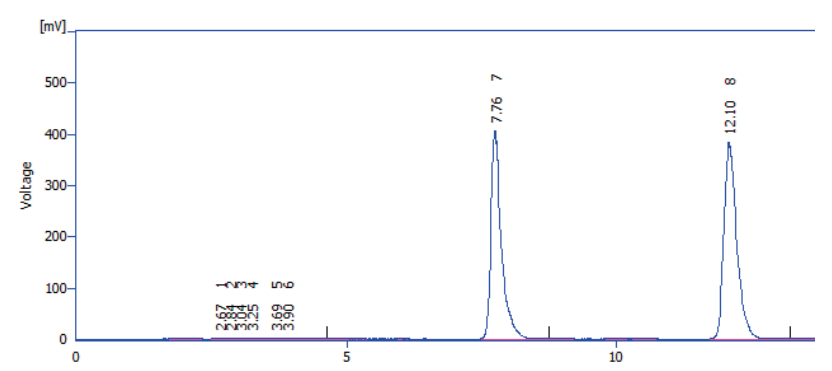

Figure 2. Sample chromatogram for the standard preparation solution containing LPZ (at $7.7 \mathrm{~min}$ ) and the internal standard (at $12.1 \mathrm{~min}$ ) according to the USP method. obtained RSD values were reasonably low, and thus reproducibility could be judged as satisfactory. However, the RSD value for the product (A) was notably higher (3.8) than the rest of the preparations, which might reflect some variation within the product. Nevertheless, all products passed the USP assay test because the amount of LPZ per label was always within the range stated by the monograph (i.e., $90-110 \%$ of label claim).

Table 2. Average USP Assay Results for Commercial LPZ Products $(n=6)$

\begin{tabular}{|c|c|c|}
\hline \multirow{2}{*}{ LPZ Capsules/Tablets } & \multicolumn{2}{|c|}{ USP Assay Test Results } \\
\cline { 2 - 3 } & Label (\%) & RSD \\
\hline A & 100.54 & 3.80 \\
\hline B & 105.94 & 0.17 \\
\hline C & 107.62 & 1.61 \\
\hline D & 106.65 & 2.30 \\
\hline E & 97.84 & 1.23 \\
\hline F & 103.26 & 2.16 \\
\hline G & 104.84 & 1.24 \\
\hline
\end{tabular}

\section{Dissolution}

The USP adoption of the rather nonselective UV method for the determination of the acid-labile drug LPZ in dissolution medium was a major motivation for this work. That is because the test result might be seriously affected by the rapid degradation of the released LPZ in the strong acidic medium. In the test, the capsule/tablet is placed in two successive media, the acid stage and the buffer stage, which are $0.1 \mathrm{M} \mathrm{HCl}$ solution and phosphate buffer (final $\mathrm{pH}$ of 6.8), respectively. Only one sample is withdrawn at the end of the acid stage ( $60 \mathrm{~min})$, and the concentration of LPZ is determined by a direct measurement of absorbance at $306 \mathrm{~nm}$ and a comparison with a similarly prepared standard solution, where no more than $10 \%$ of LPZ should have been released. The amount of LPZ released in the buffer stage was determined in the same way as the acid stage using the proper blank solution and taking the difference in absorbance between 286 and 650 $\mathrm{nm}$ instead of a single absorbance value at $306 \mathrm{~nm}$. The amount of LPZ released should not be less than $80 \%$ in the buffer stage at $60 \mathrm{~min}$.

To ensure that all obtained absorbance values from the dissolution test were in the linear response range, two calibration curves were constructed for the acidic and buffer stages covering the concentration ranges of $1.5-15 \mu \mathrm{g} / \mathrm{mL}$ and $6-30 \mu \mathrm{g} / \mathrm{mL}$, which represent release percentages of $2.5-25 \%$ and $20-100 \%$, respectively. Typical linearity equations of $y=0.017 x+0.002$ and $y$ 
$=0.029 x+0.042$ were obtained for the acid and buffer stages, respectively, with correlation coefficients of 0.999 in both cases. It should be noted, however, that at the expected values of release of less than $10 \%$ in the acid stage, the anticipated absorbance values would be quite low (less than 0.1), which, while within the linear response range, is susceptible to variation (i.e., imprecision, which might explain the high standard deviation values for the obtained percent release of products in acidic medium, as discussed later).

Results for the dissolution test applying the recommended USP UV method in acidic and buffer stages are shown in Table 3. With the exception of the product (E), which exhibited a percentage release of $13 \%$ in acid stage, all other products exhibited a percentage release significantly less than the allowed limit of $10 \%$. Therefore, it could be concluded that the product $(E)$ is the only product that might have failed the requirements of the dissolution test. It should be noted that RSD values were significantly high for the percentage release values obtained in the acid stage unlike those for the buffer stage, which is most likely a result of the very low concentrations released and the associated low values of absorbance $(0.0-0.172)$ in acidic medium. However, it is expected that if the percentage release for a product reached the specified limit of $10 \%$, a high enough absorbance would be recorded and the variation, as given by RSD, would be minimized. Thus it might be argued that the high variation observed would not actually affect the final decision regarding the percentage released since it is most significant at the very low percentages released (i.e., far from the limit of $10 \%$ ).

Table 3. Average Percentage LPZ Release $(n=6)$ Using the USP Method $(U V)^{a}$

\begin{tabular}{|c|c|c|c|c|}
\hline \multirow{2}{*}{$\begin{array}{c}\text { Product } \\
\text { Tested }\end{array}$} & \multicolumn{4}{|c|}{ Average Percentage LPZ Released } \\
\cline { 2 - 5 } & \multirow{2}{*}{ Acid Stage $^{b}$} & \multicolumn{3}{|c|}{ Buffer stage } \\
\cline { 3 - 5 } & & $30 \mathrm{~min}$ & $60 \mathrm{~min}$ & $90 \mathrm{~min}$ \\
\hline A & $0.58(0.96)$ & $103.1(10.3)$ & $102.9(7.4)$ & $99.3(3.9)$ \\
\hline B & $1.80(1.33)$ & $94(8.8)$ & $103.9(6.1)$ & $103.1(2.8)$ \\
\hline C & $0.95(0.38)$ & $98.6(2.6)$ & $104.9(1.5)$ & $104.7(1.7)$ \\
\hline D & $3.41(1.49)$ & $98.2(9.2)$ & $99.3(7.4)$ & $101.2(6.4)$ \\
\hline E & $13.0(2.5)$ & $84.6(6.6)$ & $94.5(5.7)$ & $93.9(5.0)$ \\
\hline F & $3.74(1.16)$ & $90.6(6.2)$ & $97.9(3.9)$ & $93.2(3.5)$ \\
\hline G & $1.14(1.01)$ & $92.7(3.2)$ & $99.1(4.8)$ & $101.4(4.9)$ \\
\hline
\end{tabular}

${ }^{a}$ Note that USP requires measurement at $60 \mathrm{~min}$ only.

${ }^{b} R S D$ or $S D$ values shown in parentheses.

For the buffer stage, all products satisfied the USP requirements (i.e., release of greater than $80 \%$ at 60 min). Although the USP requirements do not include sampling at other than $60 \mathrm{~min}$, results in Table 3 show the percentage release for different products after 30,60 , and $90 \mathrm{~min}$ of dissolution. Differences in the performance of the products could best be seen at $30 \mathrm{~min}$, while at later times (60 and $90 \mathrm{~min}$ ), all products achieved maximum release near $100 \%$. According to data in Table 3 and at $30 \mathrm{~min}$ of dissolution, while one product released as high as $103.1 \%$, another showed only $84.6 \%$ release (i.e., $85-$ $103 \%)$. That range became narrower when percentage release was evaluated at $60 \mathrm{~min}$ (95-105\%). Thus it might be advisable to evaluate dissolution at 30 min instead of $60 \mathrm{~min}$ for the purpose of comparing release properties of different preparations.

\section{Dissolution Test Applying HPLC Method}

Dissolution samples for all products in the two stages were assayed using an HPLC method in addition to the USP-recommended UV method described earlier. The HPLC method was based on that recommended by the USP monograph for the assay of LPZ capsules/tablets. Only the sample preparation step was modified to suit the nature of the dissolution sample in terms of the analyte as well as the $\mathrm{pH}$ of the medium (acidic $\mathrm{pH} 1.2$ or buffer $\mathrm{pH}$ 6.8). Exact details for sample preparation were discussed earlier. A standard solution was also prepared to contain the internal standard 4-ethoxyacetophenone, which is the same internal standard recommended by the USP procedure for the quantitative assay test. As part of validation of the modified HPLC method, calibration curves were prepared for each of the acid and buffer stages and linearity examined. For the acidic stage, concentrations in the range of $1-30 \mu \mathrm{g} / \mathrm{mL}$ were used, which correspond to $1.67-50 \%$ release of the theoretical ideal content. For the buffer stage, concentrations in the range of $1-30 \mu \mathrm{g} / \mathrm{mL}$ were used, which correspond to $3-100 \%$ release of the theoretical ideal content. Typical calibration equations for acidic and buffer stages were $y=0.020 x-0.024\left(R^{2}\right.$ $=0.976)$ and $y=0.032 x-0.025\left(R^{2}=0.994\right)$, respectively. Other validation parameters of precision and accuracy at low and high concentrations were also satisfactory.

The obtained average percentage released for all products tested using the HPLC method (Table 4) satisfied USP requirements for dissolution (i.e., release of not less than $80 \%$ in the buffer stage at $60 \mathrm{~min}$ and not more than $10 \%$ in the acid stage). However, one product (E) released the lowest percentage of LPZ as it released $75 \%$ and $87 \%$ at 30 and $60 \mathrm{~min}$, respectively. That is consistent with the observation that product $(\mathrm{E})$ was the only one that released greater than $10 \%$ in the initial acid stage (i.e., a larger amount of the drug was exposed for a longer time to the acidic environment with the consequent 
higher percentage of degradation). The obtained percentage release for the product $(E)$ at 60 min using the HPLC method was significantly lower $(87.3 \%)$ than that obtained by the UV method (94.5\%). The observed low percentage released here could not be simply attributed to a lower-than-expected content of LPZ in the preparation because it was concluded to be satisfactory according to USP assay results discussed earlier (97.84\%). However, it could be explained in terms of degradation of the drug in the acidic medium and the selective nature of the HPLC method, which selectively determined only LPZ, while the nonselective UV method determined both LPZ and its potential degradation product. If the absorptivity of the degradation product at the measuring wavelength was significantly different from that of LPZ, then a serious error in the estimated percentage release might be anticipated when employing the UV method (15). To further investigate potential interferences with the UV method, the absorption characteristics of LPZ and its degradation product were investigated. A solution of LPZ was prepared at a concentration near the limit of $10 \%$ release from one capsule in the employed acidic dissolution medium, which is $15 \mu \mathrm{g} / \mathrm{mL}$ in $0.1 \mathrm{M} \mathrm{HCl}$, and spectra recorded over time (Figure 3 ).

Table 4. Average Percentage LPZ Release $(n=6)$ Using HPLC Method ${ }^{a}$

\begin{tabular}{|c|c|c|c|c|}
\hline \multirow{2}{*}{$\begin{array}{c}\text { Product } \\
\text { Tested }\end{array}$} & \multicolumn{4}{|c|}{ \% LPZ Released } \\
\cline { 2 - 5 } & \multirow{2}{*}{ Acid Stage $^{b}$} & \multicolumn{3}{|c|}{ Buffer stage } \\
\cline { 3 - 5 } & & $30 \mathrm{~min}$ & $60 \mathrm{~min}$ & $90 \mathrm{~min}$ \\
\hline A & $<1$ & $103.1(10.3)$ & $103.0(7.4)$ & $99.3(3.9)$ \\
\hline B & $<1$ & $105.7(10.3)$ & $109.2(3.8)$ & $103.7(2.3)$ \\
\hline C & $<1$ & $95.1(3.5)$ & $103.5(3.9)$ & $99.4(6.7)$ \\
\hline D & $<1$ & $93.5(5.6)$ & $94.5(3.9)$ & $90.2(6.7)$ \\
\hline E & $3.7(0.71)^{b}$ & $75.4(7.9)$ & $87.3(6.5)$ & $83.4(4.8)$ \\
\hline F & $<1$ & $90.6(6.2)$ & $97.9(3.9)$ & $93.2(6.5)$ \\
\hline G & $<1$ & $93.9(5.3)$ & $101.0(6.7)$ & $100.8(7.7)$ \\
\hline
\end{tabular}

${ }^{a}$ Note that USP requires measurement at $60 \mathrm{~min}$ only.

${ }^{b} R S D$ or $S D$ values shown in parentheses.

The overlaid UV spectra show maximum absorption $\left(\lambda_{\max }\right)$ at $338 \mathrm{~nm}$ that progressively decreased with time while the minimum absorption at about $260 \mathrm{~nm}$ increased, suggesting degradation was taking place. The $306 \mathrm{~nm}$ wavelength (which was recommended by USP as a $\lambda_{\max }$ ) was an isobestic point (but not $\lambda_{\max }$ ) at which the absorbance remains almost constant with time, despite other clear changes in the obtained spectra (Figure 3); this reflects the occurrence of degradation. Therefore, although degradation was taking place, the absorbance

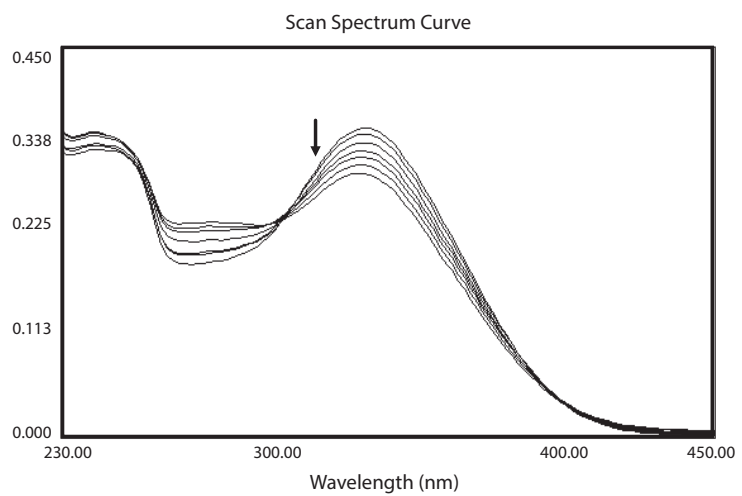

Figure 3. Overlaid UV spectra of $L P Z$ solution $(15 \mu \mathrm{g} / \mathrm{mL})$ recorded at 10-min intervals (time increases according to the direction of the arrow). Note the isosbestic point at $306 \mathrm{~nm}$ where the absorbance does not change despite degradation.

value at $306 \mathrm{~nm}$ did not change, and consequently, the calculated concentration of the dissolved drug would still be correct. Therefore, the USP method appears to be more appropriate in this context than a selective HPLC method, because in dissolution experiments, the main concern would be the overall amount of drug dissolved regardless of whether it remained chemically intact in the medium. These findings demonstrate that borderline products such as product $(E)$ might be inappropriately rejected using a selective HPLC method like the one recommended by the $B P$, but accepted using the rather nonselective UV method recommended by the USP. A $T$-test was performed to check for a significant difference between the results obtained from the two methods (HPLC and UV) at both stages (acidic, 60 min; buffer, 60 min) for all products. Results for this test are listed in Table 5. For the acidic stage, the two methods produced statistically different results where only one out of the seven products tested showed similar results, with the values obtained by the HPLC method being generally less than those of the UV method. At least in part, this could be explained as a result of the low precision of the UV method at very low absorbance values encountered when only a low percentage of LPZ is released. For the buffer stage, however, where the error in the UV method was minimized due to the release of a significant amount of drug, the results obtained by both methods are almost similar with the exception of one product (E), which repeatedly showed a significant release of LPZ in the acidic stage and consequently degraded, leading to significant differences in the results obtained by the UV and HPLC methods. Thus, for products that do not release a significant percentage of the drug in the acidic stage with the consequence of no significant degradation of LPZ, no significant difference between 
the results of the HPLC or UV methods is expected. Nevertheless, this work demonstrates with an actual example of a commercially available product that the analytical methodology employed might affect the final conclusion. A solid understanding of the principles of pharmacopeial analytical procedures is important. For example, an analyst who does not understand the role of the isosbestic point as a wavelength of detection in LPZ in a UV quantification method might not fully appreciate the danger of changing the wavelength of measurement.

Table 5. T-Test Values and $p$ Values (in parentheses) for Comparing the Two Methods (HPLC and UV) at the Two Stages ${ }^{a}$

\begin{tabular}{|c|c|c|}
\hline Product & Acidic Stage at 60 min & $\begin{array}{c}\text { Buffer Stage } \\
\text { at } 60 \mathrm{~min}\end{array}$ \\
\hline A & $1.8011(0.0906)$ & $1.2341(0.2349)$ \\
\hline B & $3.3104(0.0079)$ & $1.7295(0.1144)$ \\
\hline C & $6.1115(0.00011)$ & $0.663(0.5219)$ \\
\hline D & $4.3164(0.0015)$ & $1.4305(0.1831)$ \\
\hline E & $8.4876(<0.00001)$ & $2.3665(0.0395)$ \\
\hline F & $5.4384(0.0003)$ & $0.0624(0.9514)$ \\
\hline G & $2.7601(0.0201)$ & $0.5391(0.6028)$ \\
\hline
\end{tabular}

${ }^{a}$ Calculated at $p<0.05$.

\section{CONCLUSION}

All products tested satisfied the USP quantitative assay requirement for drug content. The selective chromatographic USP assay procedure was adapted and validated for the analysis of dissolution samples of LPZ in acidic and buffer media. The data obtained when applying both the nonselective UV method recommended by the USP together with the adapted, more selective HPLC method, leads to the conclusion that the recommended USP method (UV method) is more reliable and can give the actual percentage released, while the HPLC method reflects only the percentage of LPZ that remains intact. Thus, the more selective HPLC method will show a lower percentage released than what is actually released for products that release significant percentage of LPZ in the acid stage. Some of the released LPZ may degrade rapidly in the medium so that the degraded portion may not be accounted for by the HPLC method, although it is really a part of the dissolved amount. Only one commercial product (E) failed to satisfy the USP requirements for dissolution. The rather preliminary data presented should encourage further research to confirm our findings. Perhaps it is appropriate to recommend to other regulatory authorities who still adopt selective HPLC methods for dissolution evaluations of LPZ products (e.g., the $B P$ ) to revise their monographs.

\section{ACKNOWLEDGMENT}

The authors wish to thank the Deanship of Academic Research at The University of Jordan for financial support.

\section{CONFLICT OF INTEREST}

No conflict of interest has been declared by the authors.

\section{REFERENCES}

1. Lansoprazole Monograph. In The United States Pharmacopeia and National Formulary USP 36-NF 31; The United States Pharmacopeial Convention, Inc.: Rockville, MD, 2013.

2. Lansoprazole Monograph. In British Pharmacopoeia; The Stationary Office: London, 2013.

3. Satoh, H. Discovery and Development of Proton Pump Inhibitors. In Proton Pump Inhibitors: A Balanced View; Chiba, T., Malfertheiner, P., Satoh, H., Eds.; Frontiers of Gastrointestinal Research, Vol. 32; Karger: Basel, 2013; pp 1-17. DOI: 10.1159/000350624.

4. Gupta, H. P.; Saini, K.; Dhingra, P.; Pandey, R. Study of Acid Catalyzed Reactions of Proton Pump Inhibitors at D.M.E. Port. Electrochim. Acta 2008, 26 (5), 433448. DOI: 10.4152/pea.200805433.

5. Wahbi, A.-A. M.; Abdel-Razak, O.; Gazy, A. A.; Mahgoub, H.; Moneeb, M. S. Spectrophotometric determination of omeprazole, lansoprazole and pantoprazole in pharmaceutical formulations. J. Pharm. Biomed. Anal. 2002, 30 (4), 1133-1142. DOI: 10.1016/S0731-7085(02)00464-8.

6. Basavaiah, K.; Ramakrishna, V.; Anil kumar, U. R.; Somashekar, B. C. Spectrophotometric determination of lansoprazole in pharmaceuticals using bromatebromide mixture based on redox and complexation reactions. Eclética Quím. 2007, 32 (1), 57-64. DOI: 10.1590/S0100-46702007000100008.

7. Devi, P. U.; Krishna, K. M. Visible Spectrophotometric Determination of Lansoprazole in Pure and Pharmaceutical Formulations. Am. J. PharmTech Res. 2013, 3 (2), 2249-3387.

8. Moustafa, A. A. M. Spectrophotometric methods for the determination of lansoprazole and pantoprazole sodium sesquihydrate. J. Pharm. Biomed. Anal. 2000, 22 (1), 45-58. DOI: 10.1016/S0731-7085(99)00275-7.

9. Prasanna Kumar Reddy, B.; Ramanjaneya Reddy, Y.; Ramachandran, D. Determination of Pantoprazole Sodium and Lansoprazole in Individual Tablet Dosage Forms by RP-HPLC using single mobile phase. E-J. Chem. 2009, 6 (2), 489-494. DOI: 10.1155/2009/502472.

10. Luo, Y.; Xu, L.; Xu, M.; Feng, J.; Tang, X. A validated, specific, stability-indicating HPLC method for 
determination of lansoprazole enteric capsules and related impurities. Asian J. Pharm. Sci. 2012, 7 (2), 149-154.

11. Kumar, S. M.; Kumar, D. S.; Rajkumar, T.; Kumar, E. U.; Geetha, A. S.; Diwedi, D. Development and validation of RP-HPLC method for the estimation of lansoprazole in tablet dosage form. J. Chem. Pharm. Res. 2010, 2 (6), 291-295.

12. Patel, B.; Dedania, Z.; Dedania, R.; Ramolia, C.; Vidya Sagar, G.; Mehta, R. S. Simultaneous Estimation of Lansoprazole and Domperidone in Combined Dosage Form by RP-HPLC. Asian J. Res. Chem. 2009, 2 (2), 210-212.

13. Yeniceli, D.; Dogrukol-Ak, D.; Tuncel, M. Determination of lansoprazole in pharmaceutical capsules by flow injection analysis using UV-detection. J. Pharm. Biomed. Anal. 2004, 36 (1), 145-148. DOI:10.1016/j. jpba.2004.04.015.

14. Dogrukol-Ak, D.; Tuncel, M.; Aboul-Enein, H. Y. The Determination of Lansoprazole in Pharmaceutical Preparation by capillary Electrophoresis. Chromatographia 2001, 54 (7-8), 527-530. DOI: 10.1007/BF02491212.

15. Tokumura, T.; Machida, Y. UV absorption method should not be applied for determining amoxycillin in acidic dissolution test medium. Int. J. Pharm. 2001, 228 (1-2), 1-4. DOI: 10.1016/S0378-5173(01)008377.

16. Tapia-Albarran, M.; Villafuerte-Robles, L. Assay of amoxicillin sustained release from matrix tablets containing different proportions of Carbopol 971P NF. Int. J. Pharm. 2004, 273 (1-2), 121-127. DOI: 10.1016/j.ijpharm.2003.12.019. 